\title{
Comparison of Gravity Anomalies from Recent Global Geopotential Models with Terrestrial Gravity and Airborne Gravity over Johor Region
}

\author{
Shuib Rambat ${ }^{1}$, Nazirah Mohamad Abdullah ${ }^{2 *}$, Norehan Yaacob ${ }^{1}$, Nor’ Azizi \\ Othman $^{1}$, Zainal Abidin ${ }^{3}$
}

${ }^{1}$ Malaysia-Japan International Institute of Technology, Universiti Teknologi Malaysia, 54100 Kuala Lumpur, MALAYSIA

${ }^{2}$ Faculty of Civil Engineering and Built Environment,

Universiti Tun Hussien Onn Malaysia, 86400 Parit Raja, Johor, MALAYSIA

${ }^{3}$ Faculty Geoinformation and Real Estate,

Universiti Teknologi Malaysia, 81310 Johor Bahru, Johor, MALAYSIA

*Corresponding Author

DOI: https://doi.org/10.30880/ijie.2021.13.03.009

Received 20 December 2020; Accepted 01 May 2021; Available online 06 June 2021

\begin{abstract}
Gravity anomalies can yield an indirect but extremely useful picture of lateral changes in rock composition and structural patterns especially for rapid development area such as Johor region. The gravity anomalies can be derived from Global Geopotential Model (GGM) which is one of special product from the satellite technology that able to determine high accuracy of the earth's gravity field. In this study, the gravity anomalies derived from recent GGM published by International Global Geopotential Model were compared with five other GGMs model that compromised either terrestrial or airborne or both to derive the gravity anomalies. In order to identify the best gravity model over the Johor region, two types of GGM class model has been selected for the comparisons which known as satellite only and combined class model. The result shows that the gravity anomalies de-rived from satellite only class model with up 300 spherical harmonic coefficients is the best fit model and can be used as a reference for the Johor region. The RSME for the recent GGM via satellite only were +/5.865 and $+/-3.347 \mathrm{mGal}$ for terrestrial and airborne gravity anomalies respectively compared to other GGM.
\end{abstract}

Keywords: Global Geopotential Model (GGM), gravity anomalies, terrestrial gravity, airborne gravity

\section{Introduction}

Geodesy is the field that study about the size and shape of the earth which falls under geometrical geodesy [1] while latter became one of the main topics in physical geodesy [2]. The determination of the earth's shape can be done through earth gravity field measurement. The gravity field changes across the earth as the tectonic plates are able to move around the earth surface. Hence, it is important for development area such as Johor region to take into account the distribution of the gravity field. The distribution of gravity field information is also important to geodesist, geophysics, geodynamics, oceanographer, geologist, surveying and mapping. The instrument used to measure the gravity field are gravimeter, magnetometer or using satellite observation data, just to name a few.

The development of satellite for earth observation began in the late 50's which has revolutionized the geodesy field in several aspects including the determination of earth's gravity field [3]. In earth's gravity field, the main contribution of the satellite technology is the possibility of deriving the earth surface gravity data from the satellite information. 
The satellite information is used to derive the Global Geopotential Model (GGM). The GGM compute the spherical harmonic solutions of the earth's gravity field. During the 1960s and 1970s, GGM represent the long and medium wavelength components of the earth's gravity field [4].

However, the development of new generation satellite which has been launched specifically for gravity field determination has made the derivation more detail and accurate. Some of the satellites that were launched on the purpose of gravity field determination are Challenging Minisatellite Payload (CHAMP), Gravity Recovery and Climate Experiment (GRACE) and Gravity Field and Steady State Ocean Circulation Explorer (GOCE) [2].

CHAMP was launched in 2002 and it used satellite-to-satellite tracking (SST) in high-low mode as a measurement concept. Follow by GRACE in 2004 which used SST in low-low mode and the latest satellite is GOCE which was launched in 2009.

In this paper, six GGMs derived from GOCE, GRACE, Gravity Data and Altimetry data with different maximum degree and order had been compared in order to identify a precise and best fit ggm which been used later as a reference for determining the geoid model [5] and finally produce the gravity anomalies for the Johor region area. In addition, the GGMs also might produces geoid height or height anomaly, gravimetric geoid, deflection of the vertical gravity disturbance and gravity gradients components.

\section{Gravity Anomaly Calculation}

Gravity anomaly is the quantity left over after the effects of latitude and elevation have been removed. In geophysics, gravity anomaly can be defined as the difference between observed gravity and the field of reference model [7] while in geodetic, the gravity anomaly can be defined as difference between gravity on the geoid and normal gravity on the reference ellipsoidal [8]. In this paper, the second definition will be more appropriate.

In terms of the above definition, the gravity anomaly can be computed using Eq. (1) [8]. This equation calculates the free air anomaly or known as Faye Anomaly. The Faye anomaly is a difference between observed gravity with the reference gravity with the corrected elevation. The calculation can be done using a spread sheet such as Microsoft Office Excel.

$$
\Delta g_{F}=g_{o b s}+\partial g_{F A}-\gamma_{\phi}
$$

where $\Delta g_{F}$ is free air anomaly, $g_{o b s}$ is observed gravity (in mGal), $\gamma_{\varnothing}$ is the normal gravity, and $\partial g_{F A}$ is the free air reduction.

$\gamma_{\emptyset}$ is a prior parameter that need to be calculated before we can actually calculate the free air anomaly and the values of $\gamma_{\emptyset}$ were calculated at each station of the latitude $(\varphi)$ using Eq. (2) [7].

$$
\gamma_{\phi}=\gamma_{e} \frac{1+k \sin ^{2} \varphi}{\sqrt{1+e^{2} \sin ^{2} \varphi}}
$$

where $k$ is normal gravity constant, $e^{2}$ is the square of the first eccentricity of the ellipsoid constant, $\gamma_{e}$ is normal gravity on equator, and $\varphi$ is the latitude.

However, Eq. (1) and Eq. (2) is not considering the spherical Earth approximation which is considered adequate in physical geodesy. Thus, to present the earth more accurately, the use of an oblate ellipsoid is more preferable. Therefore, the second order of the Taylor expansion series for free-air gravity reduction were used to obtain an accurate free air anomaly. The second order of the Taylor expansion series has been derived by [9] as shown in Eq. (3).

$$
\partial g_{F A}=\frac{\partial \gamma}{\partial h}=\frac{2 \gamma_{e}}{a}\left(1+f+m-2 f \sin ^{2} \varphi\right) H-\frac{3 \gamma_{e}}{a^{2}} H^{2}
$$

where $\partial g_{F A}$ is free-air reduction, $\gamma_{e}$ is the normal gravity at the equator, $a$ is the semi major axis of the reference ellipsoidal, $f$ is the geometrical flattening of the reference ellipsoidal, $m$ is ratio of the gravitational and centrifugal force at the equator, $\varphi$ is the geocentric latitude of the point, and $H$ is the height of the point above mean sea level (geoid) [10].

Later, the gravity anomaly calculated from Eq. (3) were compared with the derived gravity anomaly from the GGM. The difference between the gravity anomaly and derive gravity anomaly were calculated using Eq. (4) [11] which known as residual. From the residual, the standard deviation and root-mean-square errors (RMSE) were obtained using statical analysis method. The decision of choosing the best fit GGM model for gravity anomaly derivation were based from the smallest standard deviation and the smallest RMSE.

$$
\Delta g_{\text {grav }(g g m)}=\Delta g_{g r a v}-\Delta g_{g g m}
$$


where $\Delta g_{\text {grav }(g g m)}$ is residual gravity anomaly, $\Delta g_{\text {grav }}$ is free air gravity anomaly obtained from existing terrestrial observation data and $\Delta g_{g g m}$ is gravity anomaly derived from GGM by using EGMLab software. The EGMLab software is a scientific software for determining the gravity and gradient components from the global geopotential as the model has been represented by the spherical harmonic coefficient that defines the potential of gravitational in the spectral domain [12]. The specific gravity-related information such as gravity anomaly $(\Delta g)$ can be computed using spherical harmonic coefficients as follows [4];

$$
\Delta_{g}(r, \varphi, \lambda)=\frac{G M}{a} \sum_{n=2}^{N_{\max }} \sum_{m=0}^{n}\left(\frac{a}{r}\right)^{n+1}\left(\frac{n-1}{r}\right)\left(\bar{C}_{n m} \cos m \lambda+\bar{S}_{n m} \sin m \lambda\right) \bar{P}_{n m}(\sin \varphi)
$$

where $\varphi$ is the latitude, $\lambda$ is the longitude, $r$ is the geocentric distance, $G$ is the gravitational constant, $M$ is the Earth's mass, $a$ is the semi major axis of reference ellipsoid, $n$ and $m$ are the degree and order, $N_{\max }$ is the maximum degree, $\bar{C}_{n m}$ and $\bar{S}_{n m}$ are the normalized geopotential coefficients, $\bar{P}_{n m}$ is the normalized associated Legendre function and $\gamma$ is the normal gravity at certain latitude obtained by Somigliana's formula.

\section{Data Description}

This study focussed on the area of Johor region, a state at southern Peninsular Malaysia and bordered by Malacca, Negeri Sembilan and Pahang. Johor region is the third largest state in Peninsular Malaysia after Pahang and Perak. The target area is bounded by geographical boundaries from $1.0^{\circ}$ to $3.0^{\circ}$ Northern latitudes $\left(1.0^{\circ} \leq \varphi \leq 3.0^{\circ}\right)$ and from $101.0^{\circ}$ to $105.0^{\circ}$ Eastern longitudes $\left(101.0^{\circ} \leq \lambda \leq 105.0^{\circ}\right)$. The study intrigued in determining the best GGM for the Johor region.

\subsection{Terrestrial Gravity Data}

At Johor region, 234 co location land gravity data have been collected by Department of Survey and Mapping Malaysia (DSMM). Wherein, the 234 co location points obtain from First Order Gravity Network and Second Order Gravity Network. The First Order Gravity Network data are measured using high precision relative gravimeter along with the precise levelling route at intervals of 40-50 km for the area. However, Second Order Gravity Network data measured using high precision relative gravimeter along the precise levelling route at intervals of 1-5 km and referred to First Class Order Gravity Network [13].

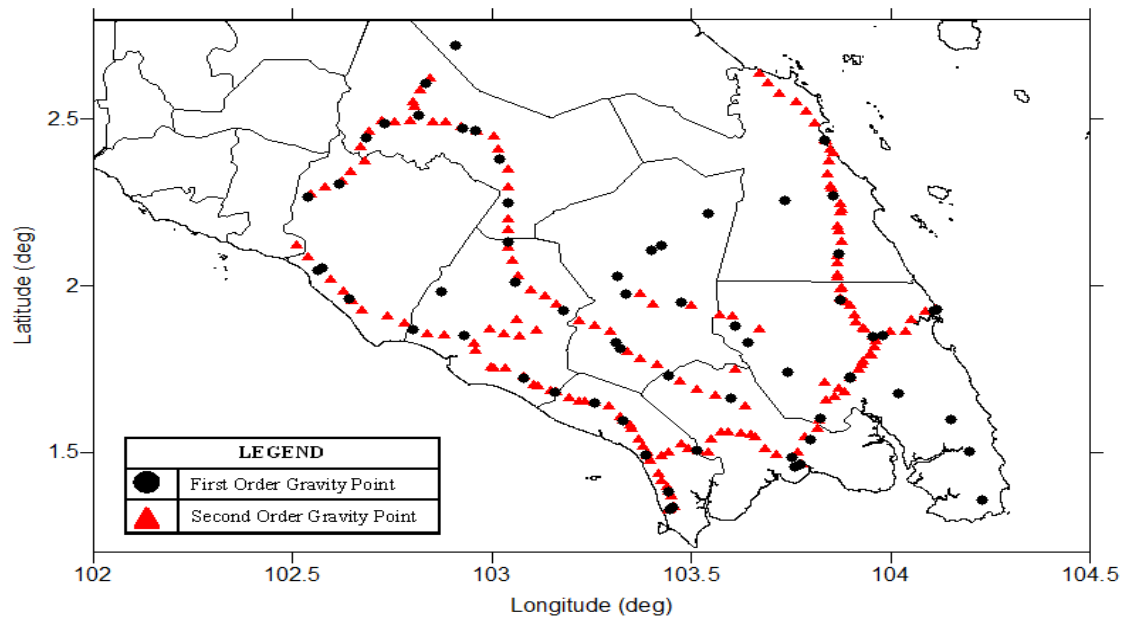

Fig. 1 - 234 co-locations of terrestrial gravity data

\subsection{Airborne Gravity Data}

The Malaysia airborne gravity survey was done on a $5 \mathrm{~km}$ resolution covering peninsular Malaysia, Sabah and Sarawak. The Malaysia airborne gravity survey was detecting a 3918 co location point cover Johor region. The airborne data system used was based on the Danish National Space Center, which is based on differential Global Positioning System (GPS) for positioning, velocity and vertical accelerations, with gravity sensed by a modified marine [4]. 


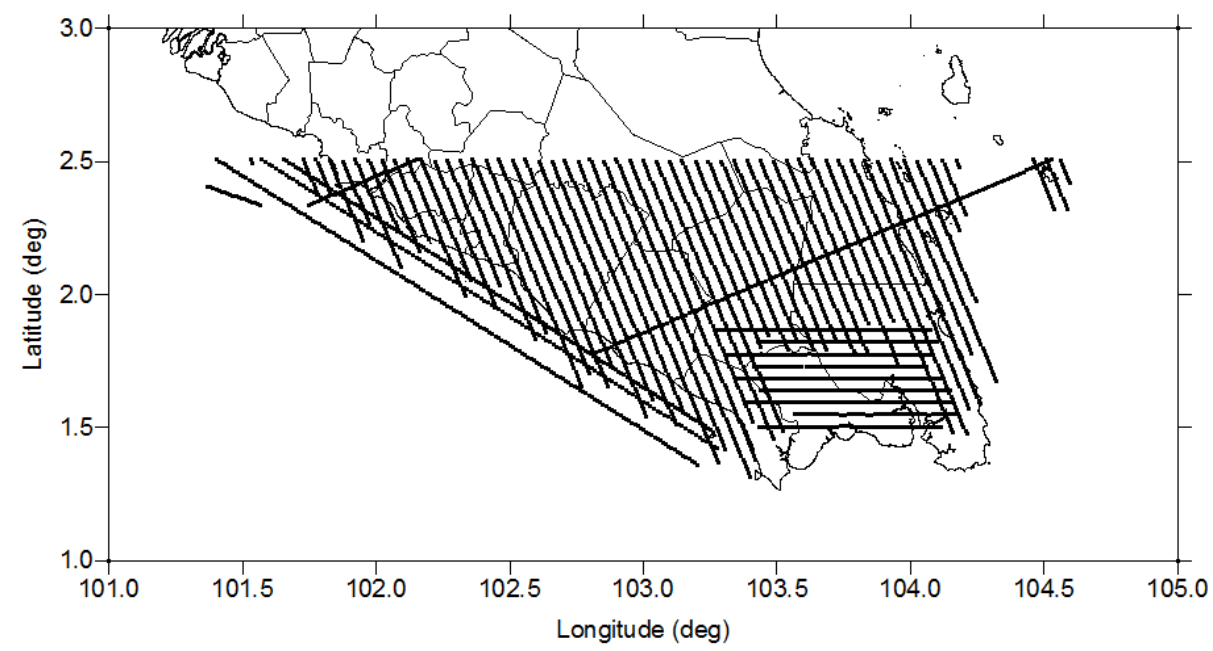

Fig. 2 - Airborne Gravity Data

\subsection{Global Geopotential Model (GGM)}

The gravity anomalies of six model of GGM were analysed and compared to determine the best fit of GGM within the Johor region. The satellite-only and combined gravity models are used in this paper. The satellite-only are derived from the analysis of the orbits of artificial Earth satellites and the combined gravity models are derived from the combination of satellite data, land, and ship-track gravity observations and marine gravity anomalies derived from satellite radar altimetry and more recently airborne gravity data [14].

The ITG_GOCE02 gravity field model was computed from 1st November 2009 until 30th Jun 2011 (7.5 months) of GOCE gradiometer and orbit data [15]. The JYY_GOCE04S model was determine from GOCE data released from 1st November 2009 until 19th October 2013. The GOGRA04S model was computed by using GOCE data released from 1st November 2009 until 19th October 2013, and GRACE data released from August 2002 until August 2009. The GO_CONS_GCF_2_DIR_R5 model was computed by using direct approach (DIR) of combination OF GOCE, GRACE, and LAGEOS orbit analysis and gradiometry [16].

The EIGEN-51C model is based on GRACE, CHAMP, terrestrial gravity and satellite altimetry information. The GIF48 model is a combination of GRACE and terrestrial gravity information, complete to degree and order 360. GIF48 was determined from a selection of 66 months spanning 2003 through 2010. The EGM2008 model is based on GRACE mission, terrestrial gravity and satellite altimetry information. This model is complete with degree and order 2159 and contained an additional spherical harmonic coefficient extending to degree 2190 [5].

Table 1 - The Six of GGM used to derive Gravity Anomalies [6]

\begin{tabular}{|c|c|c|c|c|c|c|}
\hline No. & Model & $\begin{array}{c}\text { Max. } \\
\text { Degree }\end{array}$ & $\begin{array}{c}\text { Earth Gravity } \\
\text { Constant }\end{array}$ & Radius & Years & Data Source \\
\hline \multicolumn{7}{|c|}{ Satellite-Only GGM } \\
\hline 1 & ITG_GOCE02 & 240 & $3.9860044150 \mathrm{E}+14$ & $6.37813630 \mathrm{E}+06$ & 2013 & GOCE \\
\hline 2 & JYY_GOCE04S & 230 & $3.9860044150 \mathrm{E}+14$ & $6.378136460 \mathrm{E}+06$ & 2014 & GOCE \\
\hline 3 & GOGRA04S & 230 & $3.9860044150 \mathrm{E}+14$ & $6.378136460 \mathrm{E}+06$ & 2014 & $\begin{array}{l}\text { GOCE, } \\
\text { GRACE. }\end{array}$ \\
\hline 4 & GO_CONS_GCF_2_DIR_R5 & 300 & $3.9860044150 \mathrm{E}+14$ & $6.378136460 \mathrm{E}+06$ & 2014 & $\begin{array}{c}\text { GOCE, } \\
\text { GRACE, } \\
\text { LAGEOS. }\end{array}$ \\
\hline \multicolumn{7}{|c|}{ Combined GGM } \\
\hline 5 & EIGEN-51C & 359 & $3.9860044150 \mathrm{E}+14$ & $6.378136460 \mathrm{E}+06$ & 2010 & $\begin{array}{c}\text { GRACE, } \\
\text { CHAMP, } \\
\text { G, A. }\end{array}$ \\
\hline 6 & GIF48 & 360 & $3.9860044150 \mathrm{E}+14$ & $6.37813630 \mathrm{E}+06$ & 2011 & GRACE, G, A. \\
\hline 7 & EGM2008 & 2190 & $3.9860044150 \mathrm{E}+14$ & $6.37813630 \mathrm{E}+06$ & 2008 & GRACE, G, A. \\
\hline
\end{tabular}




\section{Result and Analysis}

The analysis was based on standard deviation and Root Mean Square Error (RMSE). The standard deviation represents a precision of the observation while the RSME was refer as accuracy of observation [17]. The accuracy can be defined as the closeness of agreement between a test result and the accepted reference value. The precision is the closeness of agreement between independent test results obtained under stipulated conditions [18]. The smallest standard deviation and RMSE will representing a higher accuracy and precision of the GGM.

The statistical information for the difference of gravity anomalies observed from terrestrial gravity data and gravity anomalies computed from GGM models as shown at Table 2. The smallest standard deviation and RMSE of the residual gravity anomalies for terrestrial gravity data is $5.673 \mathrm{mGal}$ and $5.865 \mathrm{mGal}$, respectively. The GO_CONS_GCF_2_DIR_R5 is the lower standard deviation and RMSE compare to ITG-Goce02, JYY_GOCE04S, GOGRA04S, EIGEN-51C, GIF48, and EGM2008.

Table 2 - The statistical information of the residual gravity anomalies for terrestrial gravity data (unit: mGal)

\begin{tabular}{llccccc}
\hline \multicolumn{1}{c}{ No. } & Model & Minimum & Maximum & Mean & Standard Deviation & RMSE \\
\hline Satellite-Only GGM & & & & & \\
\hline 1 & ITG-Goce02 & -25.120 & 24.091 & 1.545 & 7.645 & 7.780 \\
2 & JYY_GOCE04S & -25.119 & 15.614 & -1.767 & 7.217 & 7.415 \\
3 & GOGRA04S & -25.163 & 15.475 & -1.823 & 7.212 & 7.424 \\
4 & GO_CONS_GCF_2_DIR_R5 & -25.986 & 15.642 & -1.533 & 5.673 & 5.865 \\
\hline Combined GGM & & & & & 8.128 \\
\hline 5 & EIGEN-51C & -33.498 & 14.492 & -3.705 & 7.250 & 8.314 \\
6 & GIF48 & -34.638 & 12.795 & -4.454 & 7.036 & 7.624 \\
7 & EGM2008 & -32.697 & 13.454 & -3.527 & 6.774 & \\
\hline
\end{tabular}

The Table 3 shows the statistical information of the difference of gravity anomalies observed from airborne gravity data and gravity anomalies computed from GGM models. Based on standard deviation and RMSE of the residual gravity anomalies for airborne gravity data, The GO_CONS_GCF_2_DIR_R5 had smallest standard deviation and RMSE value with $5.672 \mathrm{mGal}$ and $3.347 \mathrm{mGal}$ respectively.

Table 3 - The statistical information of the residual gravity anomalies for airborne gravity data (unit: $\mathbf{m G a l}$ )

\begin{tabular}{ccccccc}
\hline \multicolumn{1}{c}{ No. } & Model & Minimum & Maximum & Mean & Standard Deviation & RMSE \\
\hline Satellite-Only GGM & & & & & \\
\hline 1 & ITG-Goce02 & -23.352 & 26.613 & 1.304 & 7.307 & 5.891 \\
2 & JYY_GOCE04S & -23.089 & 26.240 & 0.163 & 6.873 & 4.015 \\
3 & GOGRA04S & -23.105 & 26.302 & 0.128 & 6.869 & 4.050 \\
4 & GO_CONS_GCF_2_DIR_R5 & -20.552 & 32.041 & 0.044 & 5.672 & 3.347 \\
\hline Combined GGM & & & & & 7.016 \\
\hline 5 & EIGEN-51C & -25.159 & 29.774 & 0.216 & 6.757 & 5.926 \\
6 & GIF48 & -26.441 & 30.111 & -0.458 & 6.271 & 4.865 \\
7 & EGM2008 & -25.463 & 30.143 & -0.219 & & \\
\hline
\end{tabular}

The GO_CONS_GCF_2_DIR_R5 model shows as higher accuracy and precision model to be use in Johor region. The GO_CONS_GCF_2_DIR_R5 model was computed by using direct approach (DIR) of combination of GOCE, GRACE, and LAGEOS orbit analysis and gradiometry [16]. The model used data from $1^{\text {st }}$ November 2009 until $20^{\text {th }}$ October 2013.

Table 4 represents all the residual gravity anomalies for terrestrial gravity data and airborne gravity data. The residual gravity anomalies for terrestrial gravity data is around $25.000 \mathrm{mGal}$ until $-35.000 \mathrm{mGal}$. While, the residual gravity anomalies for airborne gravity data is around $30.000 \mathrm{mGal}$ until $-25.000 \mathrm{mGal}$. 
Table 4 - The geographical distribution of the residual gravity anomalies for terrestrial and airborne gravity data






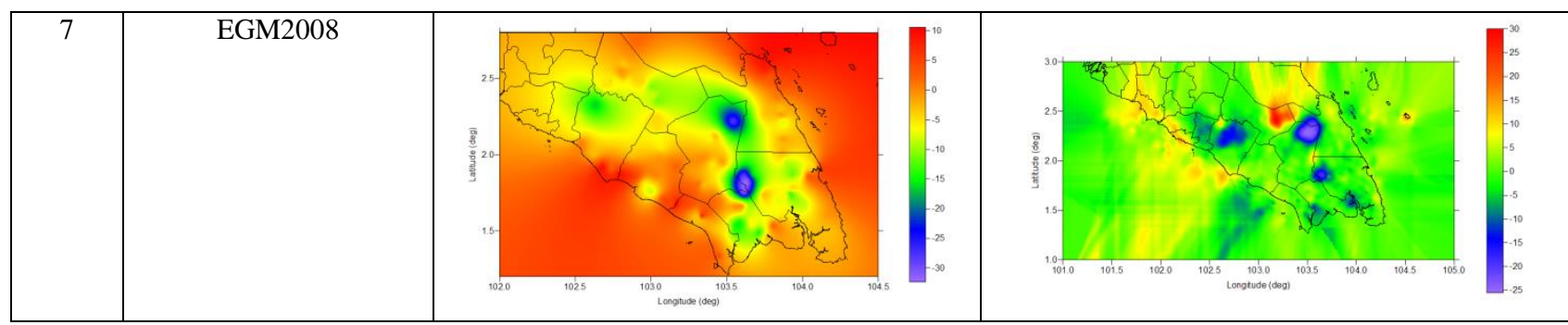

\section{Conclusion}

The paper analysed and compared gravity anomalies of recent GGMs with gravity anomalies from terrestrial gravity and airborne gravity for Johor region. Six GGM model was tested such as ITG-Goce02, JYY_GOCE04S, GOGRA04S, GO_CONS_GCF_2_DIR_R5, EIGEN-51C, GIF48, and EGM2008. The GO_CONS_GCF_2_DIR_R5 as shown in Table 4 represent as the best fit model over Johor region was processed up to 300 spherical harmonic coefficients. Gravity anomalies can yield an indirect but extremely useful picture of lateral changes in rock composition and structural patterns especially for rapid development area such as Johor region. The gravity anomalies can be derived from Global Geopotential Model (GGM) which is one of special product from the satellite technology that able to deter-mine high accuracy of the earth's gravity field. In this study, the gravity anomalies derived from recent GGM published by International Global Geopotential Model were compared with five other GGMs model that compromised either terrestrial or airborne or both to derive the gravity anomalies. In order to identify the best gravity model over the Johor region, two types of GGM class model has been selected for the comparisons which known as satellite only and combined class model. The result shows that the gravity anomalies de-rived from satellite only class model with up 300 spherical harmonic coefficients is the best fit model and can be used as a reference for the Johor region. The RSME for the recent GGM via satellite only were +/- 5.865 and +/- 3.347 $\mathrm{mGal}$ for terrestrial and airborne gravity anomalies respectively compared to other GGM.

\section{Acknowledgement}

The authors gratefully acknowledge the financial support provided by Universiti Teknologi Malaysia through Grant Tier 1 no. Q.K130000.2543.15H73. Also, would like to show our gratitude to the Center of Applied Geomatics and Disaster Prevention (CAGeD), and Faculty of Civil and Environmental Engineering, Universiti Tun Hussein Onn Malaysia for sharing their pearls of wisdom during the course of this research.

\section{References}

[1] Rapp R. (1991). Part 1. Lecture Notes, Department of Geodetic Science and Surveying. The Ohio State University.

[2] Hofmann-Wellenhof B. \& Moritz H. (2005). Physical Geodesy.Springer

[3] Balmino G. (2011). Satellite Gravity Method. Encyclopedia of Solid Earth Geophysics. Springer Netherlands, pp. 504-512

[4] Kiamehr R. \& Eshagh M. (2008). EGMlab, a scientific software for determining the gravity and gradient components from global geopotential models. Earth Science Informatics, 1(2), 93-103

[5] Sulaiman S. A., Talib K., Md Wazir M. A. \& Mohd Yusof O. (2011). Comparison of gravity anomalies from terrestrial gravity and recent geopotential models over West-Malaysia. IEEE International Conference System Engineering Technology, pp. 183-187

[6] Barthelmes F. \& Köhler W. (2016). Global Gravity Field Models. International Centre for Global Earth Models (ICGEM). http://icgem.gfz-potsdam.de/ICGEM/

[7] Mikhail K. K. (2011). Gravity Anomalies, Interpretation. Encyclopedia of Solid Earth Geophysics. Springer Netherlands, pp. 456-461

[8] Heiskanen W. A. \& Moritz H. (1967). Physical Geodesy. Freeman and Co

[9] Featherstone W. \& Dentith M. (1997). A geodetic approach to gravity data reduction for geophysics. Computers and Geosciences, 23(1), 1063-1070

[10] Sulaiman S. A. (2016). Gravimetric Geoid Model Determination for Peninsular Malaysia Using Least Squares Modification of Stokes. Universiti Teknologi Mara

[11] Omar K. M. \& Nordin S. (2010). Geodetic Practices in Malaysia. Malaysia. Universiti Teknologi Malaysia

[12] Tugi A., Din A. H. M., Omar K. M., Mardi A. S., Som Z. A. M., Omar A. H., Yahya N. A. Z. \& Yazid N. (2016). Gravity anomaly assessment using GGMS and airborne gravity data towards bathmetry estimation. International Conference on Geomatic and Geospatial Technology, XLII-4/W1, 287-297

[13] DSMM, JUPEM Geoportal (2015). Jabatan Ukur dan Pemetaan Malaysia. https://www.jupem.gov.my/ 
[14] Amos M. J. \& Featherstone W. E. (2003). Comparisons of Recent Global Geopotential Models with Terrestrial Gravity Field Data Over New Zealand And Australia. Geomatics Research Australasia, pp. 1-19

[15] Schall J., Eicker A. \& Kusche J. (2014). The ITG-Goce02 gravity field model from GOCE orbit and gradiometer data based on the short arc approach. Journal of Geodesy, 88(4), 403-409

[16] Yi W., Rummel R \& Gruber T. (2013). Gravity field contribution analysis of GOCE gravitational gradient components. Studia geophysica et geodaetica, 57(2), 174-202

[17] Bruno A. W. \& Joslin L. M. (2005). The concepts of bias, precision and accuracy, and their use in testing the performance of species richness estimators, with a literature review of estimator performance. Ecography, 28, 815829

ISO 5725-1:1994 (2016). Accuracy (Trueness and Precision) of Measurement Methods and Results (Part 1: General Principles and Definitions). The International Organization for Standardization 